\title{
El juego escolar, como método de enseñanza, permitió adquirir conocimientos y destrezas para la prevención de Dengue
}

\section{Objetivo}

Evaluar una técnica de juego "Jugando en salud: dengue" como táctica de aprendizaje para favorecer la incorporación de escolares en tareas de control y prevención de dengue.

Diseño

Estudio descriptivo cuasi-experimental. ${ }^{\star}$

Lugar

Municipio de Girardot. Estado de Aragua. Venezuela.

\section{Pacientes}

Participaron 621 escolares de 8-16 años distribuidos en grupos de estudio y control, seleccionados en 9 de 29 escuelas estatales (3.330 alumnos) mediante muestreo sistemático al azar. ${ }^{*}$ Fueron agrupados en 3 salones: salón 1 y 2 (estudio) y salón 3 (control), correspondiendo 210, 196 y 215 niños en cada grupo respectivamente.

\section{Intervención}

Previa explicación de los objetivos a los docentes del grupo estudio, se distribuyó en el salón 1 el material teórico y la técnica del juego; y en el salón 2 solo material teórico. El salón 3 mantuvo actividades habituales.

Se practicó el juego tres veces por semana durante 60 días. Consistió en 20 tarjetas azules con comportamientos, 40 amarillas con preguntas fáciles, 40 rojas con preguntas difíciles, 6 peones y una ruleta. Se realizaron evaluaciones iniciales y finales con cuestionarios validados, puntaje y escala de Likert*, para aceptación del juego, conocimientos y destrezas.

\section{Resultados principales}

Se evaluaron 597 alumnos, 309 niñas y 312 niños (relación 1:1), con una media de 11,8 años. Hubo ausencias, incorporación de 13 alumnos en el salón $\mathrm{N}^{\circ} 1$ posterior al inicio de la prueba y faltante de evaluación final en el salón 2 de una escuela. La aceptación fue alta o muy alta en el $86,7 \%$ entre los participantes del juego. Los conocimientos sobre el dengue y las destrezas (conjunto de habilidades adquiridas) medidos antes de aplicar el programa previsto fueron inferiores (6,5 y 18,4 puntos, respectivamente) a los obtenidos en la prueba final ( 8,25 y 22,9 puntos, respectivamente; $P<0,05)$. Los conocimientos tuvieron aumentos significativos en los salones 1 y 2 (Ver tabla). Las destrezas también fueron significativamente mayores que las del grupo de control $(P<0,05)$.

Tabla. Resultados principales por grupo

\begin{tabular}{c|c|l|c|l|l|c}
\multirow{2}{*}{ Conocimientos } & \multicolumn{6}{|c}{ Puntuación } \\
\cline { 2 - 7 } & \multicolumn{2}{|c|}{ Salón 1 } & \multicolumn{2}{c|}{ Salón 2 } & \multicolumn{2}{c}{ Salón 3 } \\
\cline { 2 - 7 } & $\mathbf{n}$ & Media & $\mathbf{n}$ & Media & $\mathbf{n}$ & Media \\
\hline Inicial & 197 & 6,13 & 196 & 6,09 & 215 & 7,13 \\
\hline Final & 210 & 9,28 & 174 & 8,24 & 213 & 7,33 \\
\hline $\mathbf{P}$ & \multicolumn{2}{|c|}{$<0,05$} & $<0,05$ & $>0,05$ \\
\hline
\end{tabular}

\section{usiones}

La aplicación del juego como metodología de aprendizaje en una comunidad escolar, facilitó la adquisición de conocimientos y destrezas para la prevención de dengue.

Fuente de financiamiento: no referida.

\section{Comentario}

El incremento de casos de dengue con test de ELISA positivo, confirmados mediante seroconversión y la aparición de serotipo 3 hasta mayo de $2004,^{1-4}$ nos hace recapitular sobre la necesidad de implementar estrategias costo-efectivas que reditúen en cambios de conductas sanitarias en las comunidades afectadas, especialmente las del noreste de Argentina en el caso de nuestro país. Como lo muestra el trabajo precedente, la aplicación de metodologías contructivistas con actividades lúdicas en programas educativos, como medio de adquisición de prácticas sobre temas de interés o problemas comunitarios, ha sido demostrada en la prevención de enfermedades en Latinoamérica. ${ }^{5}$ Mediante este proceso es posible lograr la formación de promotores comunitarios de salud, en este caso niños, incorporando cambios de conductas no sólo en la prevención de dengue sino de otras enfermedades.

Si bien la intervención del estudio aplicó la técnica de juego a un grupo etario amplio, la que se debe adecuar a intereses y motivaciones según las etapas evolutivas y niveles de aprendizaje diferentes, fue mayoritariamente aceptada y además permitió incrementar conocimientos y destrezas en forma concreta y a corto plazo.

Dra. Gloria F. Vega Córdova. [ Médica de Familia. Primer Nivel de Atención. Provincia de Salta. ]
La OMS $(1993)^{6}$ prioriza estimular la capacidad de acción a nivel comunitario para dengue. En nuestras poblaciones, donde los recursos diagnósticos y terapéuticos para esta enfermedad son costosos o escasos, la educación sigue siendo una herramienta válida como recurso para facilitar la acción sanitaria.

Se torna necesario definir que el impacto de las medidas de prevención primaria está determinado por las decisiones políticas, educativas y sanitarias, basadas en implementación de programas de capacitación docentes- equipo de salud, así como por el compromiso y la participación comunitaria.

\section{Conclusiones del comentador:}

La técnica lúdica escolar es un medio efectivo y eficaz para la incorporación de conductas sanitarias preventivas para dengue en las comunidades afectadas, sin olvidar que es válido aplicarlas para la promoción y prevención de otras enfermedades re-emergentes.

Vega Córdova G. El juego escolar, como método de enseñanza, permitió adquirir conocimientos y destrezas para la prevención de Dengue. Evid actual. pract. ambul. 2005;8;7. Comentado de: Un juego como estrategia educativa para el control de Aedes aegypti en escolares venezolanos. Rev Panam Salud Pública. 2003;14(6):394-401. Vivas E, Guevara de Sequeda M. PMID: 14769156

\section{Referencias}

1. Boletín epidemiológico No 13. MSP. Pcia. De Salta. Año 2004.

2. Rangeon G, Avilés G. "Respuesta serológica secundaria en la epidemia de dengue 1998 en Salta. Argentina donde co-circulan otros flavivirus". Medicina Bs. As. Vol 61.N⒉2001.61:129-136.

3. Rangeon,G. ,Avilés G. "Vigilancia de laboratorio frente a Dengue en Argentina". Despachos. Emerging Infectious Diseases CDC Vol 9 No6. Junio 2003.

4. Sistema Nacional de vigilancia epidemiológica. Ed. Servicios de salud.. Vol. 19 №30. Mexico 2002

5. Melet N. "Una estrategia metodológica para la enseñanza de la educación para la salud". Valencia. Venezuela. Ed. Universidad de Carabobo.1988.

6. OMS. Asamblea Mundial de la salud. Resolución sobre prevención y control de dengue. Año 1993. 